\title{
Avaliação de Diferentes Arquiteturas de Redes Neurais Recorrentes na Previsão do Nível de Atividade de Abelhas
}

\author{
Pedro A. B. Gomes ${ }^{1}$, Eduardo C. de Carvalho ${ }^{2}$, Helder M. Arruda ${ }^{2}$, \\ Paulo de Souza ${ }^{3}$, Gustavo Pessin ${ }^{1,2}$ \\ ${ }^{1}$ Instituto de Ciências Exatas e Naturais \\ Universidade Federal do Pará (UFPA) - Belém, PA, Brasil \\ ${ }^{2}$ Laboratório de Computação Aplicada \\ Instituto Tecnológico Vale - Belém, PA, Brasil \\ ${ }^{3}$ Data61, CSIRO - Sandy Bay, Tasmania, Australia \\ pedroabg@ufpa.br, eduardo.carvalho@pq.itv.org, helder.arruda@itv.org \\ paulo.desouza@data61.csiro.au, gustavo.pessin@itv.org
}

\begin{abstract}
Resumo. Um terço dos alimentos consumidos pela humanidade são provenientes da ação das abelhas. Estes insetos também são fundamentais para a reprodução das plantas e estão desaparecendo do planeta. Um entendimento sobre o seu comportamento, aqui abordado sobre o ponto de vista do seu nível de atividade, pode ajudar a detectar situações adversas e até potencializar o uso das abelhas em culturas. Frente a isso, neste trabalho são avaliadas seis arquiteturas de Redes Neurais Recorrentes, alternando topologia e estruturas GRU e LSTM, para prever o nível de atividade de abelhas baseado nos valores de níveis passados. Mostra-se também como o modelo com menor erro é melhorado quando treinado com mais informações de níveis anteriores.
\end{abstract}

\section{Introdução}

As abelhas são consideradas o grupo mais eficaz de insetos polinizadores pois elas necessitam das plantas para retirar o seu alimento [Gullan and Cranston 2008]. Ao contrário de outros grupos de insetos, tanto as abelhas adultas quanto suas larvas e pupas alimentamse exclusivamente de recursos florais. Por isso, para suprir sua necessidade alimentar, as abelhas visitam uma grande variedade de flores colhendo o pólen (fonte de proteína) e o néctar para a produção do mel. A atividade de polinização é, portanto, uma ação involuntária dos polinizadores, mas essencial à vida das plantas, que se utilizam de cheiros, cores e sabores para atraí-los [Cetapis-Ufersa 2017]. Além disso, de todo o alimento consumido pela humanidade, estima-se que 35\% depende da ação das abelhas [Message et al. 2012]. Isto quer dizer que cerca de um terço dos alimentos que chegam às mesas das pessoas são provenientes da ação desses insetos exercendo o papel de polinizadores.

No entanto, apesar do crescimento global do número de colmeias domesticadas, a quantidade de abelhas vem diminuindo nos Estados Unidos desde a década de quarenta, e em alguns países da Europa desde a década de sessenta [Potts et al. 2009]. Segundo [JS Pettis et al. 2013], grande parte desse declínio populacional das abelhas é proveniente de uma combinação de pesticidas e fungicidas que contamina o pólen coletado pelas abelhas para alimentar as colmeias. 
Levando isso em consideração, vê-se a importância de preservar e entender as atividades das abelhas. Assim, é fundamental estudar técnicas que poderiam ser usadas para prever a situação desses insetos. Por exemplo: (1) há plantas que oferecem flores masculinas e femininas em determinados horários. Se uma colmeia instalada em uma área registra grande movimentação neste período, já seria um forte indício de que estas abelhas estariam polinizando na cultura; (2) se a agitação das abelhas não estiver de acordo com o nível previsto, pode significar que há uma alteração no ambiente da colmeia; (3) através do horário de maior atividade registrado, pode-se deduzir que horas as plantas da cultura próxima a colmeia estão aptas à polinização.

Em [Almeida 2008], outros quesitos também são analisados, como a frequência de voo e sua influência no comportamento do enxame. Já em relação a classificação de comportamento animal, [Schwager et al. 2006] utiliza o método de aprendizado de máquina K-means para separar o comportamento de bovinos em duas classes. Dessa forma, os autores conseguem discriminar quando estes animais estão em um estado de atividade ou inatividade. No trabalho de [Martens and Sutskever 2011] os autores estudam como treinar e usar Redes Neurais Recorrentes (RNNs) [Rumelhart et al. 1986] para problemas em que os dados aparecem de forma sequencial e têm certa dependência dos dados anteriormente treinados. E no artigo de [Junyoung Chung and Bengio 2014], são estudadas as estruturas de unidade de recorrência para RNNs, GRU e LSTM, na resolução de problemas sequenciais.

Diante do exposto, este trabalho visa a predição do nível de atividade de abelhas, que pode ser abordado como um problema de previsão de séries temporais. Aqui serão usadas RNNs para prever tal comportamento baseado nos níveis de atividade passados. Diferentes arquiteturas de RNNs serão avaliadas, tal como diferentes opções de unidade de recorrência. O restante desse artigo se divide da seguinte forma: na seção 2 explica-se como os dados foram coletados e como foram criados, testados e treinados os modelos de RNNs; Os resultados são apresentados na seção 3 assim como mais testes com o melhor modelo encontrado são analisados. Por fim, a seção 4 apresenta as conclusões deste trabalho e sugere possibilidades de trabalhos futuros.

\section{Métodos}

\subsection{Coleta de Dados}

A etapa de coleta de dados foi realizada como parte do projeto Microssensores [de Souza et al. 2017] [Arruda 2015] onde etiquetas eletrônicas foram coladas em abelhas a fim de aprimorar o conhecimento sobre o comportamento desses insetos. Os dados de movimentação de abelhas foram obtidos por meio do emprego de etiquetas RFID (Radio-frequency identification) em oito colmeias, como a da Figura 1. Considerou-se o período de 1 a 31 de agosto de 2015, no qual 1.280 abelhas tiveram etiquetas afixadas ao tórax, sendo 40 abelhas por colmeia (8 colmeias) por semana (4 semanas). Cada passagem de uma abelha sobre o sensor gera um registro de movimentação que, após filtrados, geraram um total de 127.758 atividades (aproximadamente 100 registros de atividade por abelha durante o período do experimento).

O nivel de atividade de abelhas é definido neste trabalho como o total de movimentos em determinada hora, dividido pelo número de abelhas vivas naquele momento. Dessa forma, o número varia de 0,0 a aproximadamente 2,0. Zero significa que nenhuma 


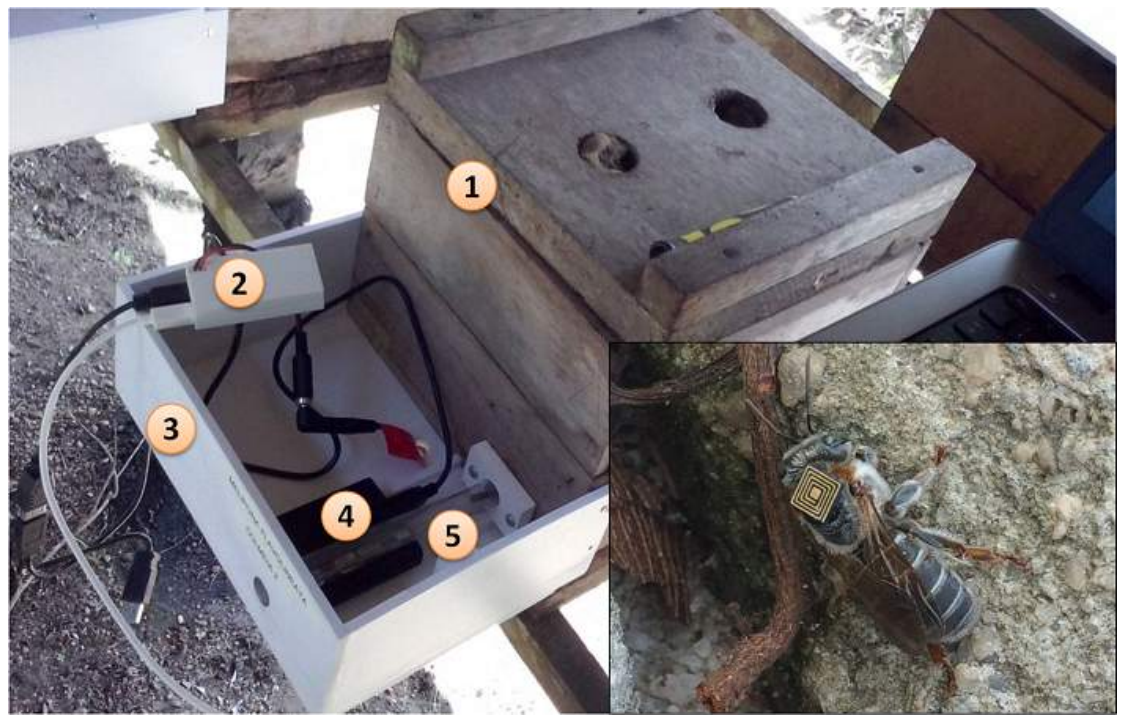

Figura 1. (1) Colmeia de Melipona Fasciculata, (2) Intel Edison para controle da antena leitora de RFID e armazenamento dos dados, (3) Caixa de PVC para armazenamento dos itens eletrônicos, (4) Antena leitora de RFID, (5) Tubo plástico para passagem das abelhas. No canto inferior direito, vê-se uma abelha com etiqueta de RFID presa junto ao tórax.

abelha está realizando atividade; dois significa que cada abelha está realizando dois movimentos naquela hora. Em média, tinha-se entre 240 e 320 abelhas vivas por dia durante o experimento. A Figura 2 mostra parte da série temporal do nível de atividade.

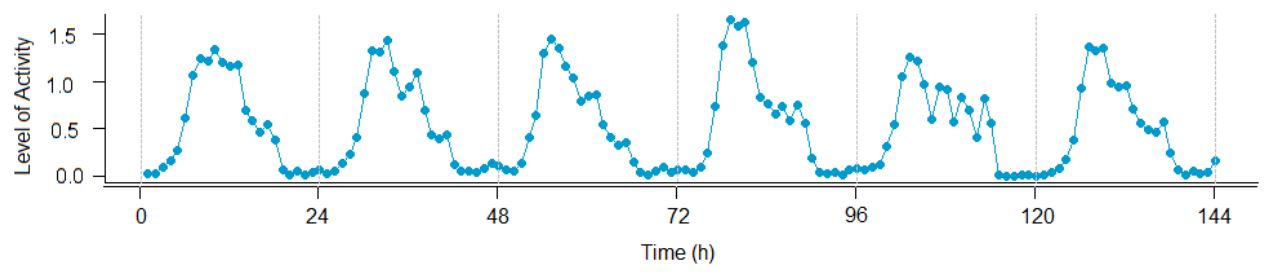

Figura 2. Série temporal parcial de atividade de abelhas dos dias 1 a 6 de agosto de 2015. Valores por hora. A série temporal de atividade de abelhas apresenta 0 número médio de atividades realizadas por cada abelha ativa no sistema.

\subsection{Criação do Modelo}

Redes Neurais Artificiais (RNAs) são um método de aprendizado de máquina que se baseia em uma metáfora do comportamento do cérebro. São formadas por unidades de processamento simples denominados neurônios que são responsáveis pelo cálculo de determinadas funções matemáticas. Os neurônios ficam organizados em uma ou mais camadas e interligados por conexões [Braga et al. 2000]. A arquitetura utilizada na rede neural depende do tipo de problema que se propõe a resolver. As RNAs com apenas uma camada são suficientes para resolver problemas linearmente separáveis, já as redes com mais camadas permitem resolver problemas não lineares. Sendo assim, as redes neurais têm sido empregadas em uma série de problemas de predição e interpolação de variáveis, como pode ser visto em [Furquim et al. 2015], [Faiçal et al. 2016] e [Carvalho et al. 2017]. 
Por sua vez, as Redes Neurais Recorrentes (RNNs) são a família de redes neurais especializadas no processamento de dados sequenciais, isto é, uma sequência de valores $\mathbf{x}(\mathbf{1}), \mathbf{x}(\mathbf{2}), \ldots, \mathbf{x}(\mathbf{T})$. Além disso, as redes recorrentes são capazes de processar de forma prática sequências muito maiores que as redes neurais que não são especializadas em dados sequenciais. Aliás, a maioria das redes recorrentes também podem processar entradas de tamanho variado [Goodfellow et al. 2016].

Redes neurais recorrentes podem ser construídas de diversas formas. Assim como quase toda função pode ser considerada uma rede neural feedforward, basicamente qualquer função que envolva uma recorrência pode ser considerada uma RNN. De forma geral a seguinte equação representa a recorrência da rede neural:

$$
h^{(t)}=f\left(h^{(t-1)}, x^{(t)} ; \theta\right)
$$

Aqui, o termo $\mathbf{h}$ indica o estado escondido no instante $\mathbf{t}$, instante atual. $h^{(t-1)}$ indica todos os cálculos acumulados até o instante anterior. $x^{(t)}$ é o vetor de entradas atual e $\theta$ é o conjunto de parâmetros compartilhados ao longo do tempo.

A Figura 3 mostra em forma de grafo uma RNN que produz uma saída a cada instante de tempo. Também observa-se o treinamento da rede e o fluxo dos dados. O loss $\mathbf{L}$ mede o quão longe cada saída da camada o está do alvo de treino correspondente $\mathbf{y}$. Esse fator $\mathbf{L}$ pode ser, por exemplo, o erro médio quadrático. Além disso, a RNN tem as conexões das entradas para as unidades escondidas parametrizadas pela matriz de pesos $\mathbf{U}$; A conexão entre as unidades escondidas parametrizadas pela matriz de pesos $\mathbf{W}$; E a matriz $\mathbf{V}$ parametriza os valores das unidades escondidas para a saída $\mathbf{0}$.

Contudo, alguns algoritmos de treinamento convencionais como o BackPropagation Through Time (BPTT) [Werbos 1987] e o Real-Time Recurrent Learning (RTRL) [Robinson and Fallside 1987] podem apresentar problemas na propagação do erro e dos valores dos instantes anteriores ao longo da estrutura, como um crescimento muito grande ou desaparecimento desses valores. Para atenuar essas situações, utiliza-se principalmente duas arquiteturas para a unidade de recorrência da rede: Long short-term memory (LSTM) [Hochreiter and Schmidhuber 1997] e Gated recurrent units (GRU) [D. Bahdanau and Bengio 2014].

Essas duas arquiteturas para a unidade de recorrência são baseadas na estrutura de portões. Por sua vez, estes controlam a quantidade da informação que entra na unidade, a quantidade que será memorizada e a informação que será passada para as próximas unidades. As duas arquiteturas têm portões diferentes que são treinados junto com os outros pesos da rede como mostrado na Figura 4.

Com o objetivo de investigar a melhor topologia de rede neural recorrente para a previsão no nível de atividade de abelhas, foram analisados 3 formatos de rede com: uma camada escondida com 4 unidades de recorrência; duas camadas escondidas com 2 unidades de recorrência cada, totalmente conectadas; e quatro camadas escondidas com uma unidade cada. Todas elas com uma camada de saída. E para cada formato, um tipo diferente de unidade GRU ou LSTM. A Figura 5 mostra essas arquiteturas. Assim, gerou-se, respectivamente seis modelos: GRU-4, GRU-2X2, GRU-1-1-1-1, LSTM-4, LSTM-2X2 e LSTM-1-1-1-1. 


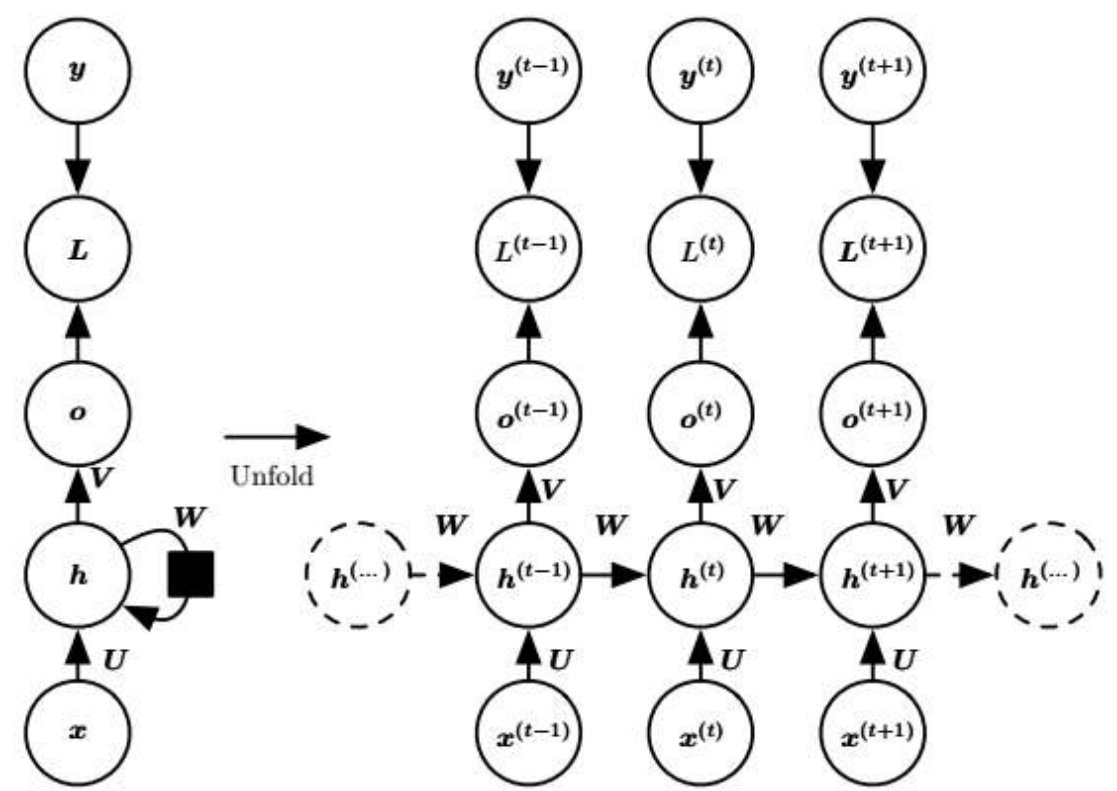

Figura 3. Grafo da rede recorrente que computa o valor do loss $L$ e através da comparação da saída $o$ gerada, e dos valores conhecidos $y$. 0 vetor de entrada é $0 x$, a rede apresenta uma saída a cada instante e os parâmetros são passados pela camada escondida. No grafo da esquerda, o quadrado preto nas setas indica a passagem do instante de tempo. O grafo da direita apresenta a mesma rede de forma expandida ao longo dos instantes de tempo [Goodfellow et al. 2016].

\subsection{Treinamento e Teste}

Os modelos foram avaliados através do Root Mean Squared Error (RMSE) calculado pela fórmula:

$$
R M S E=\sqrt{\frac{1}{n} \sum_{t=1}^{n} e_{t}^{2}}
$$

onde $e$ é a diferença entre o valor que se tem do nível de atividade e o predito pelo modelo.

Os dados foram preparados de forma que criou-se uma tabela de duas colunas, uma com o nível de atividade no instante t-1 (feature) e outra com o nível de atividade no instante $\mathbf{t}$ (target), uma hora a frente. Dito isso, os modelos têm como entrada os dados da primeira coluna e tentam prever o nível de atividade do instante seguinte.

Finalmente, para treinar e testar as redes, os dados foram embaralhados e divididos em $70 \%$ para treino e $30 \%$ para teste. Assim, para ter mais credibilidade nos resultados, cada modelo foi treinado e testado 10 vezes.

\section{Resultados}

Mediante a inicialização randômica da matriz de pesos das redes, cada arquitetura foi executada 10 vezes, como dito anteriormente. Desse modo, o gráfico da Figura 6 apresenta o erro de cada arquitetura, onde cada boxplot contém os valores das 10 execuções. Logo, observa-se que a melhor arquitetura foi a GRU-2x2, obtendo uma média de 0,214628 de RSME, sendo o mínimo de 0,207741. 


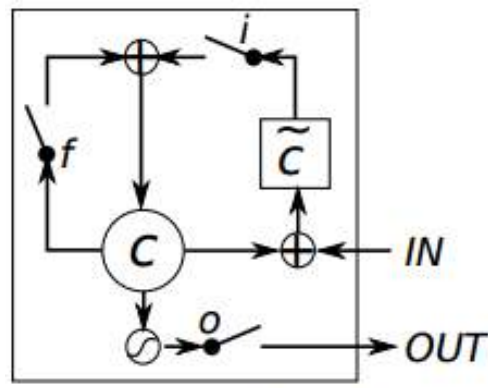

(a) Long Short-Term Memory

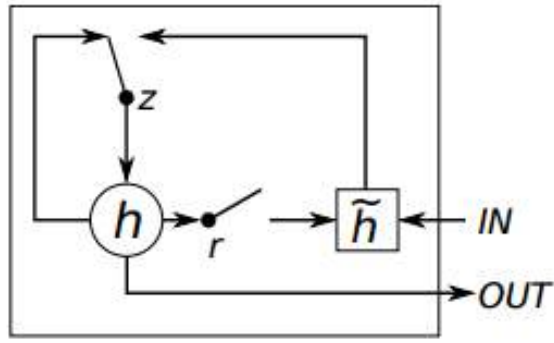

(b) Gated Recurrent Unit

Figura 4. Desenho das estruturas (a) LSTM e (b) GRU. (a) $i, f$ e $o$ são os portões input, forget e output, respectivamente. $c$ e $c \sim$ são a célula de memória e o novo conteúdo dela. (b) $r$ e $z$ são os portões reset e update. $E \boldsymbol{h}$ e $h^{\sim}$ são a ativação $\mathrm{e}$ o candidato a ativação [Junyoung Chung and Bengio 2014].

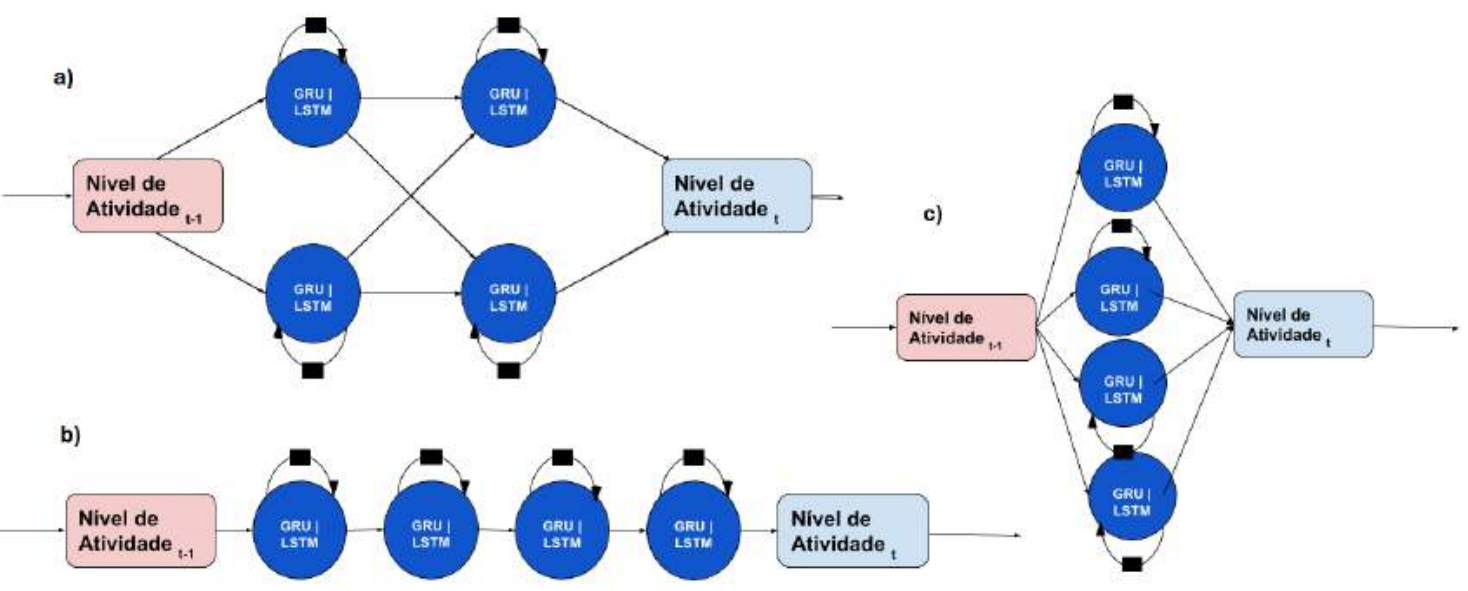

Figura 5. Desenho dos modelos criados: a) duas camadas escondidas com duas unidades cada, b) quatro camadas com uma unidade, c) uma camada com 4 unidades; Todas as estruturas possuem uma camada de saída e as unidades de recorrência ora empregam GRU, ora LSTM, totalizando 6 modelos. 0 quadrado preto nas setas indica a passagem do instante de tempo.

Analisando a melhor execução da GRU-2x2, a de RMSE igual a 0,207741, observa-se pelas Figuras 7 e 8 como os valores preditos acompanham os valores observados. O gráfico da Figura 7 organiza os níveis de atividades observados em forma crescente assim como suas respetivas predições. Nota-se que os valores preditos acompanham os observados nas 120 amostras de hora.

Já a Figura 8, também apresenta uma observação de cerca de cindo dias, em 120 horas de amostras de nível de atividade, porém de forma sequencial como foram coletadas. As amostras são do dia 22 a 27 de agosto. Observa-se que as predições conseguiram acompanhar os valores observados inclusive nos momentos de pico.

\subsection{Variação da janela de entrada}

No intuito de melhorar o resultado da previsão, foram feitos mais alguns testes com a arquitetura GRU-2x2. Entretanto, dessa fez variou-se o tamanho da janela de entrada, 


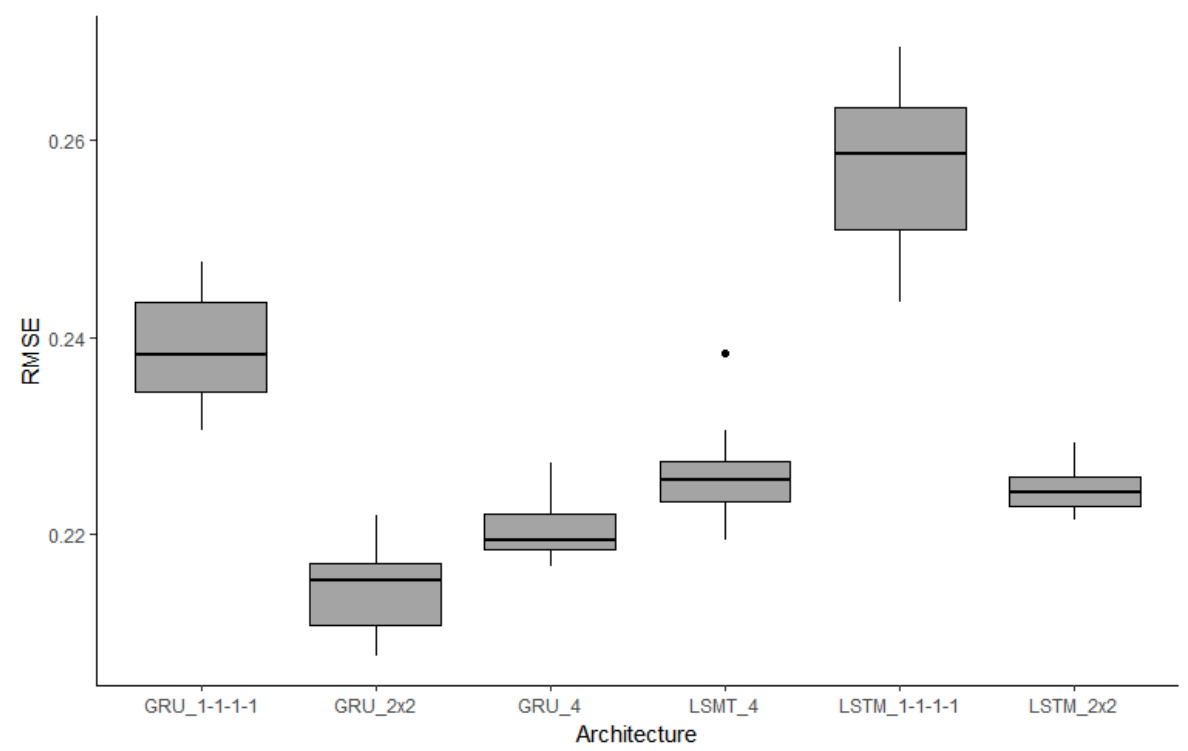

Figura 6. Cada boxplot apresenta o resultado do erro RMSE das 10 execuções de cada arquitetura.

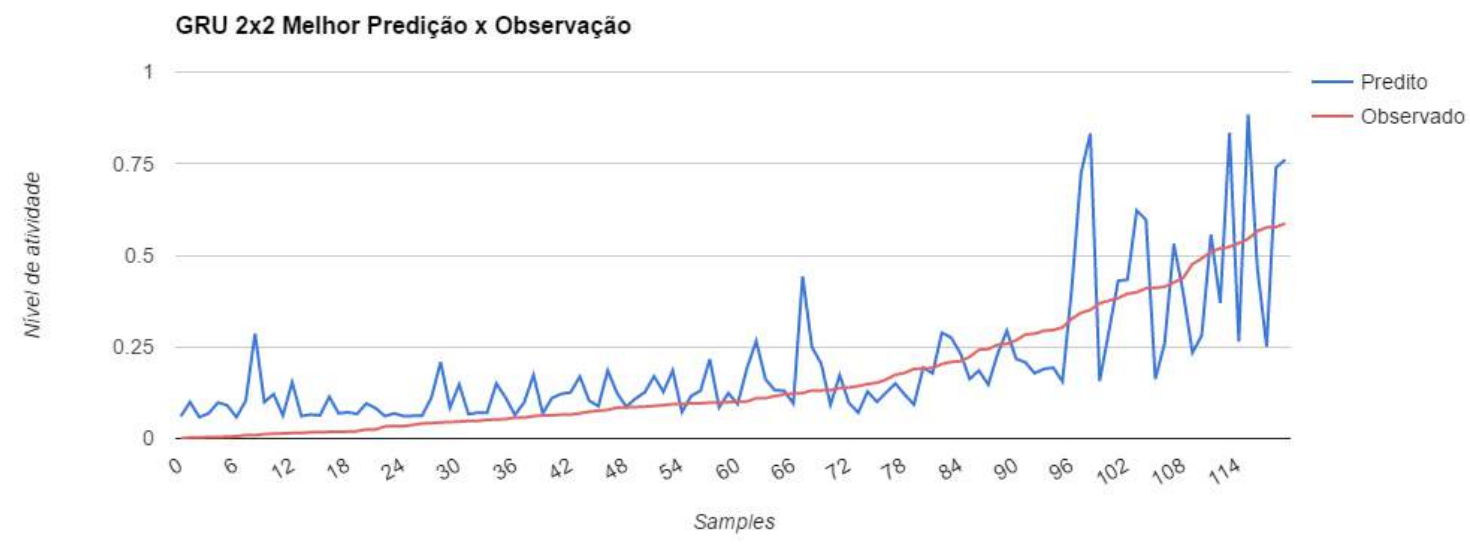

Figura 7. Amostras do nível de atividade organizadas de forma crescente acompanhadas do valor correspondente predito pela RNN GRU-2x2.

ou seja, testou-se quantidades diferentes de instantes anteriores para prever o instante seguinte, estimulando a habilidade da RNN em manter informações úteis ao longo do tempo.

Os testes foram feitos usando 3, 5, 8, 13, 21, 34 e 55 instantes de tempo anteriores às amostras preditas. A Figura 9 mostra os erros de cada configuração. Este gráfico aponta que a melhor configuração é a t-34 indicando que uma maior janela não implica sempre em redução do erro, vide t-55. Como definido na seção 2, o nível de atividade é calculado considerando o total de movimentos dividido pelo número de abelhas vivas naquela hora. Ou seja, tendo o conhecimento do nível de atividade das últimas 34 horas, pode-se estimar, com o modelo GRU-2x2, o nível no instante atual com um RMSE de 0,171735. Em outras palavras, visto que o nível variou entre 0,0 e 2,0, o erro dessa configuração foi em torno de apenas $8 \%$. 


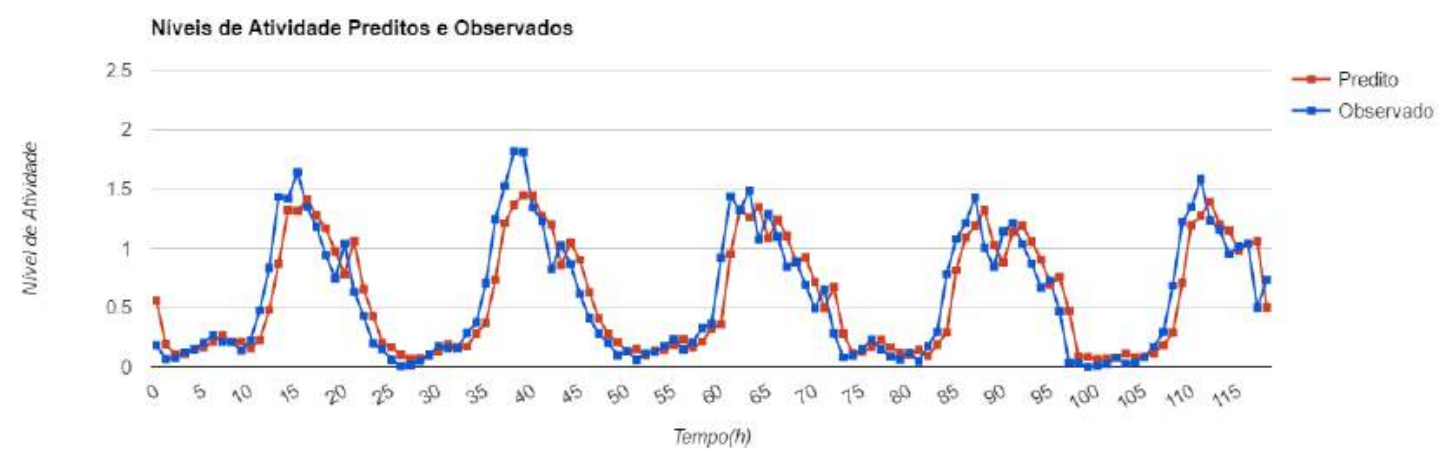

Figura 8. Cinco dias de observações e suas respectivas predições.

RMSE vs. Instantes passados de Tempo

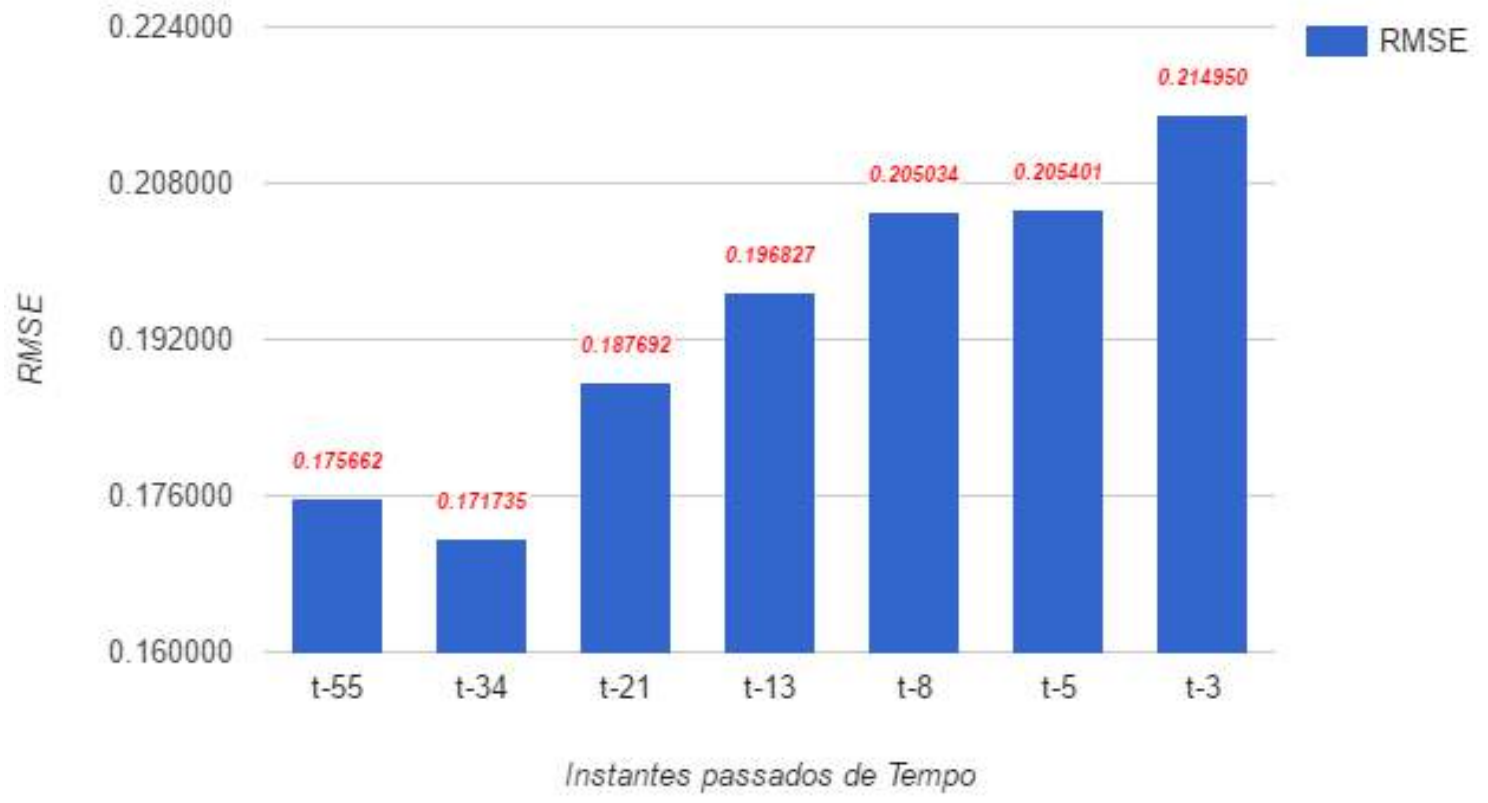

Figura 9. RMSE do modelo GRU-2x2 para cada tamanho de janela de tempo.

\section{Conclusões e trabalhos futuros}

Neste trabalho foram analisados seis modelos de redes neurais recorrentes. As configurações foram treinadas e testadas com a série temporal do nível de atividade de abelhas no intuito de prever o nível no instante seguinte. A configuração com o menor RMSE foi a GRU-2x2 que possui duas camadas escondidas com duas unidades de recorrência GRU e a camada de saída com um neurônio. Além disso, treinou-se o melhor modelo com tamanhos variados de janela temporal obtendo o erro mínimo de 0,171735 quando utilizou-se 34 registros passados, t-34.

Certamente, um melhor conhecimento sobre o comportamento das abelhas pode contribuir muito para o meio ambiente e para nossas vidas. Frente a isso, diversos trabalhos futuros podem ser elaborados. Por exemplo: (1) Análise do nível de atividade de abelhas em diferentes espécies próximas a determinada cultura para indicar a espécie mais eficiente para a polinização dessas plantas, (2) Incluir variáveis climáticas na predição, 
(3) Testar arquiteturas maiores de RNNs, e (4) Utilizar outros métodos de aprendizado de máquina para prever o nível de atividade de abelhas.

\section{Agradecimentos}

Os autores agradecem aos colegas Bruno Ferreira (ITV) e Hanna Carvalho (UFPA) pelas frutíferas discussões. Além disso, os autores agradecem ao Daniel Santiago (Embrapa) por auxílios no desenvolvimento do trabalho. O primeiro autor agradece também a CAPES pela concessão da bolsa de mestrado. Para finalizar, os autores agradecem a Vera Fonseca e a equipe do projeto microssensores (CSIRO/ITV).

\section{Referências}

Almeida, G. F. (2008). Fatores que interferem no comportamento enxameatório de abelhas africanizadas. Departamento de Biologia, Programa de Pós-Graduação em Entomologia.

Arruda, H. M. (2015). Arquitetura Computacional para Manuseio de Dados de Clima e Movimentação de Abelhas com Etiquetas Eletrônicas. Programa de Mestrado Profissional em Uso Sustentável de Recursos Naturais em Regiões Tropicais do Instituto Tecnológico Vale.

Braga, A., Ludemir, T., and Carvalho, A. (2000). Redes Neurais Artificiais: Teoria e Aplicações. LTC editora.

Carvalho, E., Ferreira, B., Ferreira, J., de Souza, C., Carvalho, H., Suhara, Y., Pentland, A., and Pessin, G. (2017). Exploiting the use of recurrent neural networks for driver behavior profiling. International Joint Conference on Neural Networks (IJCNN).

Cetapis-Ufersa (2017). Sem Abelha Sem Alimento. www . semabel ha semal imento . com.br.

D. Bahdanau, K. C. and Bengio, Y. (2014). Neural machine translation by jointly learning to align and translate. ICLR.

de Souza, P., Williams, R., Quarrelld, S., Budi, S., Susanto, F., Vincent, B., Allend, G., and Almeida, A. (2017). Agent-based modeling of honey bee forager flight behaviour for swarm sensing applications. Environmental Modelling and Software (under review).

Faiçal, B. S., Pessin, G., Pereira, G., Carvalho, A. P. L. F., Gomes, P. H., and Ueyama, J. (2016). Fine-tuning of uav control rules for spraying pesticides on crop fields: An approach for dynamic environments. International Journal on Artificial Intelligence Tools.

Furquim, G., Pessin, G., Faiçal, B. S., Mendiondo, E. M., and Ueyama, J. (2015). Improving the accuracy of a flood forecasting model by means of machine learning and chaos theory. Neural Computing and Applications, 27:1129-1141.

Goodfellow, I., Bengio, Y., and Courville, A. (2016). Deep Learning. MIT Press. http: / / www. deeplearningbook.org.

Gullan, P. J. and Cranston, P. S. (2008). Os insetos: um resumo entomológico. Tradução de Sonia Hoenen. Roca. 
Hochreiter, S. and Schmidhuber, J. (1997). Long short-term memory. Neural computation, 9(8):1735-1780.

JS Pettis, E. L., Andree, M., Stitzinger, J., Rose, R., and vanEngelsdorp, D. (2013). Crop pollination exposes honey bees to pesticides which alters their susceptibility to the gut pathogen nosema ceranae. PLoS ONE.

Junyoung Chung, Caglar Gulcehre, K. C. and Bengio, Y. (2014). Empirical evaluation of gated recurrent neural networks on sequence modeling. NIPS 2014 Deep Learning and Representation Learning Workshop.

Martens, J. and Sutskever, I. (2011). Learning recurrent neural networks with hessianfree optimization. International Conference on Machine Learning, Bellevue, WA, USA, (28).

Message, D., Teixeira, E. W., and Jong, D. D. (2012). Polinizadores no Brasil: Contribuição e Perspectivas para a Biodiversidade, Uso Sustentável, Conservação e Serviços Ambientais.

Potts, S. G., Roberts, S. P. M., Dean, R., Marris, G., Brown, M., Jones, R., and Settele, J. (2009). Declines of managed honey bees and beekeepers in europe. Journal of Apicultural Research, (49):15-22.

Robinson, A. J. and Fallside (1987). The utility driven dynamic error propagation network. Cambridge University Engineering Department.

Rumelhart, D., Hinton, G., and Williams, R. (1986). Learning representations by backpropagating errors. Nature, 323:533-536.

Schwager, M., Anderson, D. M., Butler, Z., and Rus, D. (2006). Robust classification of animal tracking data. Computers and Electronics in Agriculture, 56 (2007):46-59.

Werbos, P. J. (1987). Generalization of backpropagation with application to a recurrent gas market model. 